\title{
Interactive Energy Management for Networked Microgrids with Risk Aversion
}

\author{
Dongxiao Wang, Runji Wu, Chun Sing Lai, Xuecong Li, Xueqing Wu, Jinxiao Wei, Yi Xu, Loi Lei \\ Lai
}

\begin{abstract}
For microgrids (MGs) optimal operation, one heated topic is the uncertainty management associated with renewable variations and electricity load forecasting errors. On the other hand, the networking of MGs is receiving an increasing attention in recent years. In this paper, an interactive energy management strategy is developed for high renewable-penetrated MGs. The control method includes two steps. In the first step, a local optimization is proposed for each microgrid to minimize the operation cost during the whole scheduling periods. In the second step, a global optimization is conducted for networked microgrids. CVaR based risk averse measure is introduced here to provide a risk-hedging strategy for microgrids energy management. Formulated models are solved by the easily implemented and computationally inexpensive mix integer linear programming (MILP) solver. Case studies demonstrate the feasibility of the proposed method by identifying optimal scheduling results.
\end{abstract}

Index Terms-Energy Management, Networked Microgrids, Uncertainties, Risk aversion

\section{INTRODUCTION}

$\mathrm{U}$ NDER the pressure of fossil fuel shortage and air pollution, countries around the world are increasingly integrating the renewable energy sources (RESs) into the modern power systems, primarily in the form of solar photovoltaic (PV) panels and wind generators. Among the resources, solar capacity is around $390 \mathrm{GW}$ and wind capacity are over $500 \mathrm{GW}$ [1] . Composed by distributed generators, energy storage systems, loads and other electric components, microgrids (MGs) are emerging under such situation as a promising method to integrate renewable resources and meet end users' electricity demand. MGs can operate both in grid connected modes and islanded modes, with different operation objectives.

To further improve system reliability and preserve the customer privacy, various MGs can be connected to form a networked system. Networked MGs possess the capability of decreasing the network operation cost in grid-connected modes and reducing load shedding amount in islanded modes. The

This work was supported in part by the Department of Finance and Education of Guangdong Province 2016[202]: Key Discipline Construction Programme, China and Research funding from Guangdong Foshan Power Construction Corporation Group Co. Ltd., Foshan, China.

D. Wang, R. Wu, C. S. Lai, X. Li, and L. L. Lai are with School of Automation, Guangdong University of Technology, Guangzhou, China. C. S. Lai is also with School of Civil Engineering, University of Leeds, UK. (c.s.lai@leeds.ac.uk; 1.1.lai@gdut.edu.cn)

X. Wu, J. Wei and Y. Xu are with Guangdong Foshan Power Construction Corporation Group Co. Ltd., Foshan, China. energy management system (EMS) is essential for the operation of MGs, with the main responsibilities being to control the energy consumption and power control of a MG. By incorporating energy management strategies and optimization algorithms into the EMSs of networked MGs, they form the major components of smart distribution management systems [3]. Numerous studies have been conducted in the literature on the intelligent energy management of networked microgrids. The currently prevailing EMSs can be categorized into three types, centralized EMS [4], [5], decentralized EMS [6], and hybrid EMS [7].

On the other hand, the intermittent and stochastic characteristics of renewable energy resources bring new challenges on the scheduling of microgrids [8]. Therefore, uncertainty managements in MGs become an active research field recently, which are mainly categorized into robust optimization and stochastic optimization. The authors in [10] presented an optimal energy management strategy to satisfy the demand and supply of a grid-connected microgrid with RES penetration. A robust formulation is developed to account for the worst-case amount of harvested RES. In [11], the authors developed a scenario-based robust energy management method to accommodate renewable generation and load uncertainties in the worst case. Through optimizing the worst-case scenario, the MG robust energy management solution is derived based on Taguchi's orthogonal array method.

Stochastic optimization, as another well-known optimization tool, has been used in the planning, operation, and control of MGs. The authors in [12] proposed a two-stage stochastic programming approach to reduce the operational cost. A scenario reduction method is conducted to get reduced scenarios in the optimization process. In [13], the authors employed a finite horizon stochastic mixed integer quadratic programming model to minimize the microgrid operation cost. In [14], a stochastic framework was proposed for optimal scheduling of microgrid resources over the control period. The framework addresses uncertainties of islanding duration, and prediction errors of demand and renewable generation. Stochastic optimization brings high computational requirements with increasing number of generated scenarios. In addition, it only provides probabilistic guarantee for constraint satisfaction [15]. On the other hand, although robust optimization is immune against uncertain data sets, it could cause over-conservative operation scheduling results in MGs [15]. 
Detailed review of previous research points out some opening issues in the energy management of microgrids. Some research investigates the uncertainty management in a single microgrid, without considering about the emergence of networked microgrids. Some research solves the coordinated energy management of networked microgrids, without considering system uncertainties. To the best of authors' knowledge, few previous works consider about the risk management in the scheduling of networked microgrids. Given the uncertainties in the system, the risk value should be well considered into system optimization. Compared with the existing works, the novelty contributions and the salient features of the work are twofold:

1) Considering uncertainties in the system, a risk component is proposed to quantify system adequacy and security on a probabilistic basis. Based on the conditional value-at-risk ( $\mathrm{CVaR})$ concept, the risk value after risk aversion is proposed to represent the potential loss above the mean value;

2) A networked microgrids mechanism is employed by taking a variety of system uncertainties into account; a microgrid can sell/buy surplus/shortage power directly from other microgrids. Uncertainties in this work include load uncertainty and renewable generation uncertainty.

The remaining paper is organized as follows. Section II presents the components and configuration of networked microgrids, and introduces risk aversion definitions. Section III introduces the components modelling. Section IV presents the detailed risk-averse microgrids control model. Section V presents the numerical simulations to demonstrate the effectiveness of the proposed approach. Section VI concludes the paper.

\section{II.PROBLEM DESCRIPTION}

\section{A. Configuration of Networked Microgrids}

A typical microgrid consists of renewable generation sources, controllable distributed generators (CDGs), loads (including non-controllable load and controllable load), and battery energy storage systems (BESSs). Renewable generation resources are able to provide clean and sustainable electricity in the system, which is an important source of electricity generation. Controllable distributed generators, such as micro turbines, can provide stable electricity to the appliances in the microgrid. Battery energy storage systems are acting as an energy buffer, which can shift energy usage via its charging/discharging behavior. Controllable load can help maintaining electricity usage balance through demand response strategies. The general objective of a microgrid is to minimize its operation cost in grid connected mode, and to provide reliable electricity supply in islanded modes.

From Fig. 1, the necessary components are given in each microgrid, together with the local power flow. In this framework, each microgrid first runs a local optimization to determine the components scheduling results. Power trading among microgrids is conducted here after generating local results, so that microgrids can purchase surplus power or sell extra power generation directly with each other with lower cost. The microgrids are also able to trade with utility grid, in case of not obtaining enough power from internal components or other microgrids. By employing a networked microgrids control mechanism, optimal operation results are obtained from the whole system point of view.

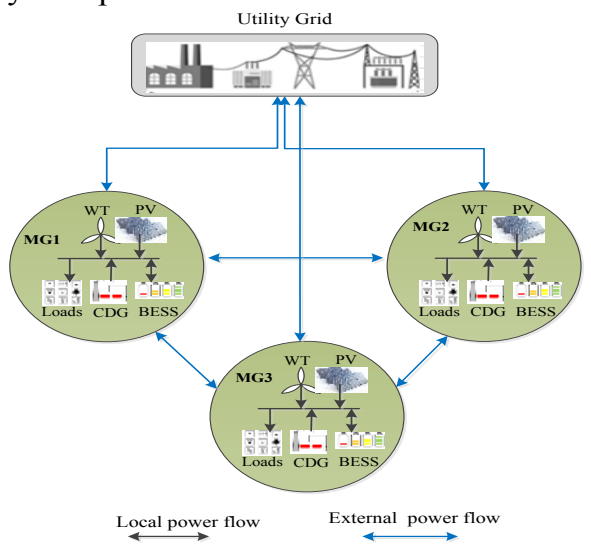

Fig. 1 Structure of networked microgrids

\section{B. Risk Aversion}

Risk is a consequence of action taken in spite of uncertainty in the system [16]. It is assumed that $f(x, y)$ is a loss representation where $\mathrm{x}$ refers to the decision variable and $\mathrm{y}$ refers to the uncertain variable. By incorporating risk measurement into the objective function, the aim is to find an optimum compromise between minimum operation cost and resilience of networked microgrids system. Value at risk (VAR) is a typical method to measure and manage risk. However, it is non-coherent risk measure with non-convexity, non-smoothness, etc., which makes it difficult to be included in optimization modelling. To avoid this problem, CVaR, also known as average value at risk, is employed in this work as an alternative risk measure. $\mathrm{CVaR}$ is defined as:

$$
C V a R=\alpha+\frac{1}{P \cdot(1-\beta)} \sum_{s=1}^{P} f\left(x, y_{s}\right)
$$

Where $\alpha$ is the value at risk, $\beta$ is the confidence level, and $P$ is the number of scenarios. Equation (1) indicates the expected conditional value of the cost function, whose value is greater than $\beta$-percentile. For linear cost function problems, minimizing $\mathrm{CVaR}$ is able to be formulated as a linear programming problem, which is quite attractive in practical applications.

\section{MODELLING OF MICROGRIDS COMPONENTS}

In this section, the various components of microgrids are explicitly given. This includes the modelling and constraints. Uncertainty modelling of renewable resources and daily load are given as well.

\section{A. $C D G s$}

Conventional distributed generators in a microgrid system can have many different forms, such as fuel cells, diesel generators, and micro turbines. In this work, the modelling of micro turbines is introduced, whose fuel cost is formulated as a linear function:

$$
C_{t}^{C G}=a^{C G}+b^{C G} P_{t}^{C G}
$$


where, $C_{t}^{C G}$ refers to the CDG operation cost at time $t ; a^{C G}$ / $b^{C G}$ are CDG cost coefficients; $P_{t}^{C G}$ is the CDG power output.

Ramp rate limits and power constraints should be satisfied for the stable operation of micro turbines, denoted as below:

$$
\begin{gathered}
\underline{P^{C G}} \leq P^{C G} \leq \overline{P^{C G}} \\
\left\{\begin{array}{l}
P_{t}^{C G}-P_{t-1}^{C G} \leq \operatorname{Ramp}_{C G}^{U p} \\
P_{t-1}^{C G}-P_{t}^{C G} \leq R a m p p_{C G}^{D o w n}
\end{array}\right. \\
\chi_{t}^{S U}+\chi_{t}^{S D} \leq 1 ; \quad \chi_{t}^{S U}, \chi_{t}^{S D} \in\{0,1\}
\end{gathered}
$$

where, $\underline{P^{C G}} / \overline{P^{C G}}$ denote CDG lower/upper limits of power output; $\operatorname{Ramp}_{C G}^{U p} / \operatorname{Ramp}_{C G}^{\text {Down }}$ are CDG ramping up/down limits; $\chi_{t}^{S U} / \chi_{t}^{S D}$ are CDG startup/shutdown indicator, where 1 represents CDG is in operation and 0 represents it is not. Power constraints are explained in Eq. (3). Ramp rate limits are given in Eq. (4). Equation (5) demonstrates that micro turbines are not allowed to startup and shutdown simultaneously.

\section{B. BESSS}

BESSs operation cost are considered in this part, which mainly refers to the maintenance cost [17]. A linear function in (6) can be used to denote the operation cost:

$$
C_{t}^{E S}=\gamma^{E S} P_{t}^{E S} \Delta t+\gamma^{E S} E_{t}^{E S} \eta^{L} \Delta t
$$

where, $C_{t}^{E S}$ is BESS operation cost; $P_{t}^{E S}$ is the charging/discharging power of BESS; $\Delta t$ is the time duration when power and energy are mutually converted; $\gamma^{E S}$ is lifetime depression coefficient of BESS; $\eta^{L}$ is leakage loss factor of BESS.

The following constraints should be met during BESS operation:

$$
\begin{gathered}
E_{t+1}^{E S}=E_{t}^{E S}-P_{t}^{E S} \Delta t-\left|P_{t}^{E S}\right| \eta^{C} \Delta t-E_{t}^{E S} \eta^{L} \Delta t \\
S O C_{t}=E_{t}^{E S} / E_{R}^{E S} \\
\underline{S O C}_{t} \leq S O C_{t} \leq \overline{S O C}_{t} \\
\bar{P}_{t}^{E S, C h r} \leq P_{t}^{E S} \leq \bar{P}_{t}^{E S, D i s} \\
\chi_{t}^{E S, D i s}+\chi_{t}^{E S, C h r} \leq 1 ; \chi_{t}^{E S, D i s}, \chi_{t}^{E S, C h r} \in\{0,1\}
\end{gathered}
$$

where, $E_{t}^{E S}$ is BESS energy at time $t ; \eta^{C}$ is charging/discharging loss factor of BESS; $S O C_{t}$ represents battery state of charge (SOC) at time $t, \underline{S O C} / \overline{S O C}$ are SOC lower/upper limits; $\chi_{t}^{E S, D i s} / \chi_{t}^{E S, C h r}$ are BESS discharging/charging indicator; $\bar{P}_{t}^{E S, D i s} / \bar{P}_{t}^{E S, C h r}$ are the upper limits of BESS discharging/charging power.

BESS capacity change is given in Eqs. (7), (8), including net energy injection, energy losses during charging/discharging process, and leakage loss. BESS SOC constraints representation is given in Eq. (9). Equation (10) denotes BESS charging/discharging power is limited within lower/upper bounds.
Equation (11) refers to BESS which is not allowed to be charged/discharged simultaneously.

\section{C.Incentive-based Demand Response Model}

This paper mainly considers about incentive-based demand response, such as direct load control, interruptible service, and emergency demand response program. Incentives will be paid to customers who are willing to increase or reduce their energy consumption when requested. The controllable load cost is represented by a linear function as [18]:

$$
C_{t}^{C L}=l_{1}+l_{2} P_{t}^{C L}
$$

where, $C^{C L}$ is the controllable load cost; $l_{1} / l_{2}$ are the intercept and slope respectively; $P^{C L}$ is the controllable load amount.

The maximum ratio of controllable load is constrained by:

$$
\begin{gathered}
\frac{P^{C L}}{P^{L}} \leq \bar{\varsigma} \\
\left\{\begin{array}{l}
P^{C L}-P_{C L}^{-} \geq 0 \\
P^{C L}+P_{C L}^{+} \leq P_{C L}^{M a x}
\end{array}\right.
\end{gathered}
$$

where, $\bar{\varsigma}$ is the controllable load maximum ratio; $P^{L}$ is the electricity load; $P_{C L}^{-} / P_{C L}^{+}$represent load decrement/increment; $P_{C L}^{M a x}$ represents the upper bound of controllable load.

\section{Uncertain Sets of RESs and Load}

RESs generation (i.e. wind power and solar power) and electricity load are regarded as uncertainties in microgrid system. Correlated scenarios are generated based on historical data, which allows the correlated probability distributions to be estimated based on the statistical correlations in the uncertainties. Based on the probability density functions (PDFs) of uncertainties, Monte Carlo simulations are employed to randomly generate scenarios. Wind power and solar power forecast errors can be modelled by the Beta distribution:

$$
f\left(\Delta P^{R E S} ; \lambda_{1}, \lambda_{2}\right)=\Delta P^{R E S \lambda_{1}-1}\left(1-\Delta P^{R E S}\right)^{\lambda_{2}-1} N
$$

where, $\Delta P^{R E S}$ refers to output forecast error of renewable output; $\lambda_{1}, \lambda_{2}$ represent the shape parameters of Beta distribution; $N$ refers to the normalization error.

Load forecast errors can be modelled by the Gaussian distribution:

$$
f\left(\Delta x ; \mu_{x}, \sigma_{x}^{2}\right)=\frac{1}{\sqrt{2 \pi \sigma_{x}^{2}}} \exp \left[-\frac{\left(\Delta x-\mu_{x}\right)^{2}}{2 \sigma_{x}^{2}}\right]
$$

where, $\Delta x$ refers to load forecast error; $\mu_{x}, \sigma_{x}^{2}$ represent mean and standard deviation.

\section{RISK-AVERSE ENERGY MANAGEMENT MODEL}

This section describes the mathematical formulation of proposed risk-averse energy management model. In step 1, local optimization is carried out by individual microgrid EMS. In step 2 , a global optimization is conducted to minimize the overall 
operation cost of networked microgrids. A 24-hour scheduling horizon is chosen in the formulation of proposed model, with one-hour uniform interval of time.

\section{A. Single Microgrid EMS}

The objective of the optimization model is to minimize the operation cost of each MG in the distribution network with system constraints satisfied, as shown below. The objective function contains $\mathrm{CDG}$ cost (i.e. its generation cost, and startup/shutdown cost), BESS maintenance cost, controllable load cost, the exchanged power cost (i.e. price of buying and price of selling).

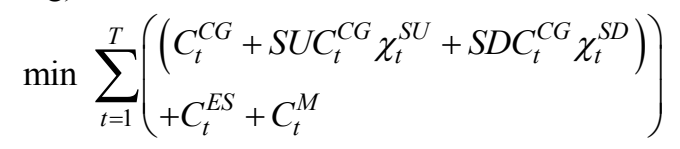

The exchanged power cost in each MG is calculated as:

$$
C_{t}^{M}=\rho_{t} P_{t}^{M}
$$

where, $\rho_{t}$ is the power price at time $t ; P_{t}^{M}$ is the exchanged power amount for the MG at time $t$. Exchange power cost is composed of price of buying and price of selling. Specifically, when $P_{t}^{M}>0, \rho_{t}$ represents the selling price, and $P_{t}^{M}$ represents the surplus power; when $P_{t}^{M}<0, \rho_{t}$ represents the buying price and $P_{t}^{M}$ represents the shortage power.

The following constraints should be satisfied in the energy management process of microgrids.

\section{1) Load balance constraints:}

For each $\mathrm{MG}$, the total power generation from renewable generators, CDGs, and BESS should be balanced with local demand and exchanged power with distribution network.

$$
P_{t}^{C G}+P_{t}^{E S}+P_{t}^{R E S}=P_{t}^{M}+P_{t}^{L}-P_{t}^{C L}
$$

2) CDG constraints:

Equations (3)-(5) are specifying the CDG operation constraints.

\section{3) BESS constraints:}

BESS operational constraints are specified in (8)-(11).

\section{4) Controllable load constraints:}

The incentive-based demand response model constraints are given in (13), (14).

After completing local optimization by the microgrid energy management system, each microgrid can determine the BESS charging/discharging status, CDGs startup/shutdown schedule, controllable load decrement/increment value, surplus/shortage power. The calculated values are communicated to the distribution system operator for global optimization.

\section{B. Networked Microgrids EMS}

After receiving the information about surplus, shortage amount from each microgrid, distribution network operator starts to conduct a global optimization for networked microgrids. It is assumed that each microgrid has a more attractive electricity rate by purchasing power directly from adjunct microgrids in the distribution network. Therefore, microgrids firstly aim to meet each other's electricity needs from adjunct microgrids, and then choose to buy power from distribution network when not enough power is provided by neighboring microgrids.

The objective of the upper level EMS is to minimize the operation cost of networked microgrids by running a global optimization.

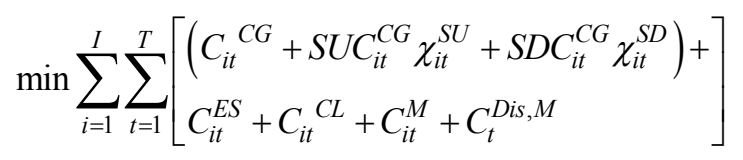

where, $i$ represents the $i^{\text {th }}$ microgrid, $t$ represents the $t^{\text {th }}$ time step. The optimization function consists of CDG cost, BESS cost, controllable load cost, microgrids internal power exchange cost, microgrids power exchange cost with distribution network.

Specially, the definitions of $C_{i t}^{M}$ and $C_{t}^{D i s, M}$ are given by:

$$
\begin{gathered}
C_{i t}^{M}=\rho_{i t} P_{i t}^{M} \\
C_{t}^{D i s, M}=\rho_{t} P_{t}^{D i s, M} \\
P_{t}^{D i s, M}=\sum_{i=1}^{I}\left(P_{t}^{C G}+P_{i t}^{E S}+P_{i t}^{R E S}+P_{i t}^{M}-\left(P_{i t}^{L}-P_{i t}^{C L}\right)\right)
\end{gathered}
$$

where, $\rho_{i t}, \rho_{t}$ refer to the microgrid internal exchange price with adjunct microgrids, and external price with distribution network respectively; $P_{t}^{\text {Dis,M }}$ is the exchanged power amount between networked microgrids and the distribution network; $I$ is the number of MGs in distribution network; $P_{i t}^{M}$ is the surplus/shortage power from microgrids, which can be calculated in local optimization.

The microgrids system uncertainties are considered in global optimization. Therefore, Eq. (20) is formulated into a probabilistic version to mitigate the risky decision making. Besides, the initial large set of scenarios is trimmed to a small number of representative scenarios in this paper to improve computational efficiency. An efficient backward method is employed to estimate the original whole scenarios and reduce the scenarios number. The risk associated with the cost variability is explicitly captured into the model through conditional value at risk. A risk-averse version of Eq. (20) can be written as:

$$
\min \sum_{i=1}^{I} \sum_{t=1}^{T}\left[\begin{array}{l}
\left(C_{i t}{ }^{C G}+S U C_{i t}^{C G} \chi_{i t}^{S U}+S D C_{i t}^{C G} \chi_{i t}^{S D}\right)+ \\
C_{i t}^{E S}+C_{i t}{ }^{C L}+C_{i t}^{M}+C_{t}^{D i s, M}+\left(w \cdot C V a R_{\beta}\right)
\end{array}\right]
$$

where, $w$ is the weighting factor for price risk, which is chosen as 10 in this paper, $C_{V a R}$ is conditional value at risk with confidence level $\beta$.

Constraints: In each scenario $k$, the following denoted constraints should be satisfied for the stable operation of networked microgrids. CDG constraints in (3)-(5), BESS constraints in (8)-(11), and controllable load constraints in (13), (14) should be satisfied, which is similar to local optimization. The distinguished parts are given below:

1) Network power balance constraints:

Network power balance constraints should be met, as shown in (23). 


\section{2) Exchanged power constraints:}

$$
\underline{P}^{E x c h} \leq P_{t}^{D i s, M} \leq \bar{P}^{E x c h}
$$

where, $\underline{P}^{E x c h} / \bar{P}^{E x c h}$ are the lower/upper limits of interconnection exchange between microgrids and distribution network.

After the global optimization is conducted, the networked EMS model can finally present the optimal BESS charging/discharging status, CDG scheduling results, microgrids internal power exchange amount, and external power exchange amount with the distribution network.

\section{V.CASE STUDIES}

The proposed approach is tested on the networked microgrids structure shown in Fig. 1, demonstrating the internal flow and external flow. All MILP-based models have been simulated on MATLAB platform with Mosek toolbox [19].

The controllable load maximum ratio is set as $25 \%$, which means a maximum $25 \%$ of daily load can be used via incentive demand response model. The intercept and slope of Eq. (12) are set as $\$ 0.28 / \mathrm{kWh}$ and 0.05 . The lower/upper limits of exchanged power with distribution network are $\pm 2000 \mathrm{~kW}$. The parameters of conventional distributed generators in each microgrid are given in Table 1 . The life-time BESS depression coefficient is 0.04 , the lower/upper limits of SOC are $20 \% / 80 \%$, and charging/discharging coefficients are 0.95 and 0.97 . Three microgrids are assumed to have the same capacity BESS with rated power at $170 \mathrm{kWh}$, maximum charging/discharging power at $125 \mathrm{~kW}$. The renewable generation and daily load are based on the day-ahead forecasted data collected from Australia Energy Market Operator [20], which is shown in Fig. 2. The power exchange price between microgrids and distribution network are given in Fig. 2 as well. The confidence level is set as 0.90 here to model the risk-averse strategy. It should be noted that the higher the confidence level is, the more different results the system has as compared with risk neutral strategy.

Table 1 Parameters of CDGs in each microgrid

\begin{tabular}{c|c|c|c}
\hline \hline Parameters & CDG1 & CDG2 & CDG3 \\
\hline$a^{C G}, b^{C G}(\$ / \mathrm{kWh})$ & $0.28,0.034$ & $0.30,0.040$ & $0.35,0.042$ \\
$\underline{P}^{C G}, \overline{P^{C G}}(\mathrm{~kW})$ & 0,220 & 0,200 & 0,180 \\
\hline $\begin{array}{c}\operatorname{Ramp}_{C G}^{U p}, \operatorname{Ramp}_{C G}^{\text {Down }} \\
(\mathrm{kW} / \mathrm{h})\end{array}$ & 75,75 & 70,70 & 80,80 \\
\hline$S_{C}^{C G}, S D C^{C G}(\$)$ & $0.32,0.16$ & $0.37,0.24$ & $0.31,0.18$ \\
\hline \hline
\end{tabular}
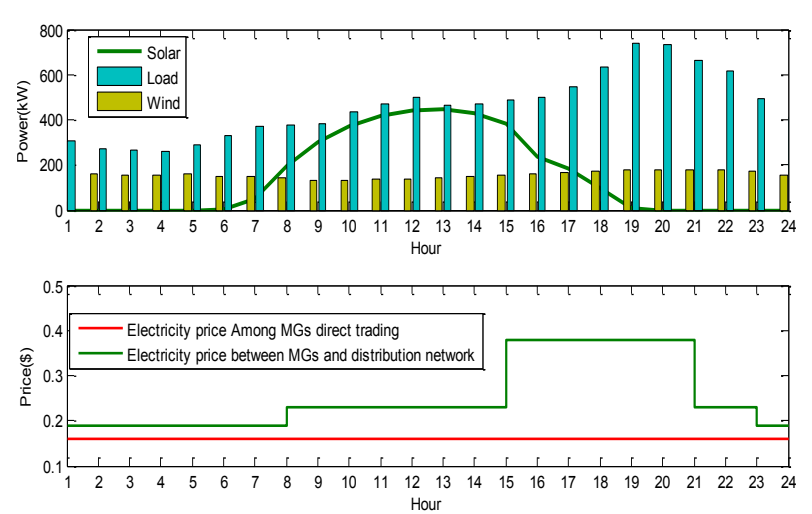

Fig. 2 Renewable generation and daily load, electricity price in MGs network

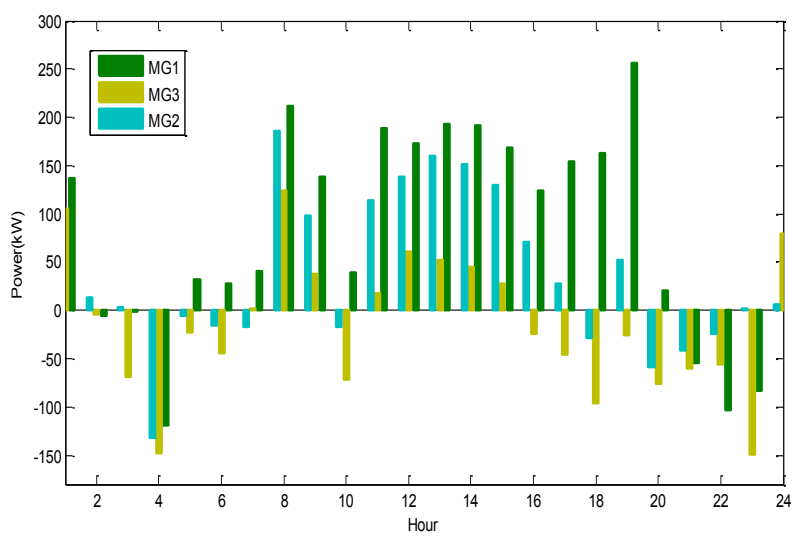

(a)Case 1 Risk neutral scenario

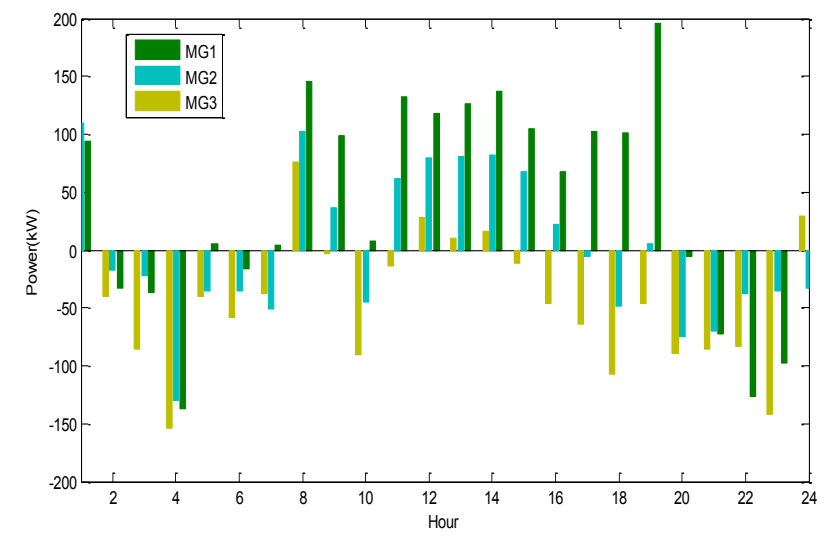

(b) Case 2 Risk averse scenario

Fig. 3 Power exchange scheduling results in three microgrids under risk neutral and risk averse scenarios

Two cases are compared to demonstrate the effectiveness of the proposed control scheme. Case 1: proposed networked microgrids control scheme with risk neutral scenario; Case 2: proposed networked microgrids control scheme with risk-averse scenario where confidence level is set to 0.90 . The simulation results are analyzed below to show the different simulation results under two cases.

Fig. 3 presents the power exchange results in each microgrid under two cases. The risk neutral scenario results are presented in Fig. 3(a), and the risk-averse scenario results are presented in Fig. 3(b). A positive value in Fig. 3 means the microgrid has surplus power, while a negative value means the microgrid has a shortfall. As observed, microgrids under risk neutral scenario have more surplus power, indicating a lower operation cost is 
expected in this case. Fig. 4 presents the BESS, controllable load and CDG scheduling results in the two scenarios. The average BESS SOC, average controllable load ratio, and overall operation cost in the microgrids under two scenarios are compared in Table 2. It can be found that risk-averse scenario has higher BESS SOC level, higher controllable load ratio, and lower operation profit as compared with risk neutral scenario.

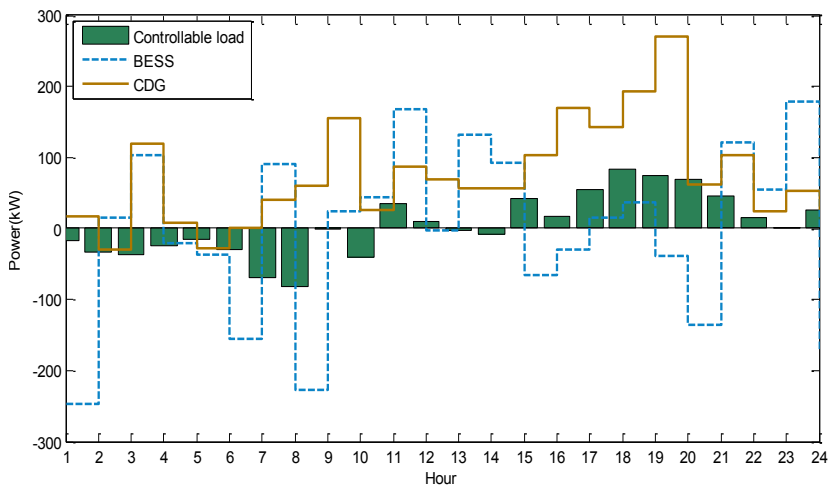

(a) Case 1 Risk neutral scenario

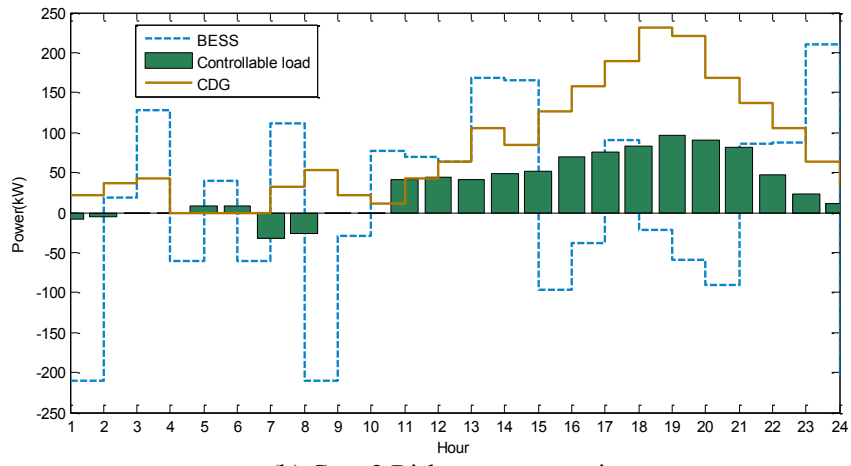

(b) Case 2 Risk averse scenario

Fig. 3 BESS, controllable load, and CDG scheduling results in three microgrids under risk neutral and risk averse scenarios

Table 2 Comparison of BESS SOC, controllable load ratio, and operation cost between risk neutral and risk averse scenarios

\begin{tabular}{c|c|c|c}
\hline \hline Scenario & Average SOC & $\begin{array}{c}\text { Average control- } \\
\text { lable load ratio }\end{array}$ & $\begin{array}{c}\text { Operation } \\
\text { cost (\$) }\end{array}$ \\
\hline Risk neutral & 0.623 & 0.124 & -160.24 \\
\hline Risk averse & 0.742 & 0.138 & -132.57 \\
\hline \hline
\end{tabular}

\section{CONCLUSION}

This paper proposes an interactive energy management strategy for networked microgrids considering renewable generation uncertainties and electricity load uncertainties. There are two steps in the energy management model, i.e. local microgrid optimization and global networked microgrids optimization. In each step, the control objective is to minimize the operation cost while satisfying equality and inequality constraints in the system. A risk-hedging mechanism is included in the energy management model via conditional value at risk measure. According to the simulation results, the proposed methodology identifies an effective scheduling plan for networked microgrids as well as providing a risk-averse strategy.

\section{REFERENCES}

[1] Renewables 2018 Global Status Report - REN21, [Online]: http://www.ren21.net/wp-content/uploads/2018/06/17-8652_GSR2018
FullReport web final .pdf (Accessed 20/03/2019)

[2] C. S. Lai, Y. Jia, L. L. Lai, Z. Xu, M.D. McCulloch, K. P. Wong, "A comprehensive review on large-scale photovoltaic system with applications of electrical energy storage", Renewable and Sustainable Energy Reviews, vol. 78, pp. 439-451, 2017.

[3] Z. Wang, B. Chen, J. Wang, M. M. Begovic, and C. Chen, "Coordinated energy management of networked microgrids in distribution system," IEEE Trans. Smart Grid, vol. 6, no. 1, pp. 45-53, Jan. 2015.

[4] Y. Xu, W. Zhang, and W. Liu, "Distributed dynamic programming-based approach for economic dispatch in smart grids," IEEE Trans. Ind. Inform., vol. 11, no. 1, pp. 166-175, Feb. 2015.

[5] N. Nikmehr and S. N. Ravadanegh, "Optimal power dispatch of multi-microgrids at future smart distribution grids," IEEE Trans. Smart Grid, vol.6, no. 4, pp. 1648-1657, Jul. 2015.

[6] N. O. Song, J. H. Lee, H. M. Kim, Y. H. Im, and J. Y. Lee, "Optimal energy management of multi-microgrids with sequentially coordinated operations," Energies, vol. 8, no. 8, pp. 8371-8390, Aug. 2015.

[7] Z. Wang, B. Chen, and J. Kim, "Decentralized energy management system for networked microgrids in grid-connected and islanded modes," IEEE Trans. Smart Grid, vol. 7, no. 2, pp. 1097-1105, Mar. 2016.

[8] C. S. Lai, Y. Tao, F. Xu, W. W. Y. Ng, Y. Jia, H. Yuan, C. Huang, L. L. Lai, Z. Xu, and G. Locatelli, "A robust correlation analysis framework for imbalanced and dichotomous data with uncertainty," Information Sciences, vol. 470, pp. 58-77, 2019.

[9] Y. Wang, S. Mao, and R. M. Nelms, "On hierarchical power scheduling for the macrogrid and cooperative microgrids," IEEE Trans. Ind. Informat., vol. 11, no.6, pp. 1574-84, Dec. 2015.

[10] Y. Zhang, N. Gatsis, G. B. Giannakis, "Robust energy management for microgrids with high-penetration renewables," IEEE Trans. Sustain. Energy, vol. 4, no. 4, pp. 944-953, Oct. 2013.

[11] Y. Xiang, J. Liu, and Y. Liu, "Robust energy management of microgrid with uncertain renewable generation and load," IEEE Trans. Smart Grid, vol. 7, no. 2, p. 1034-1043, March 2016.

[12] A. S. Farsangi, S. Hadayeghparast. M. Mehdinejad, and H. Shayanfar, "A novel stochastic energy management of a microgrid with various types of distributed energy resources in presence of demand response programs", Energy, vol. 160, pp. 257-274, vol. 160, Oct. 2018.

[13] Y. Zhang, F. Meng, R. Wang, W. Zhu, and X. Zeng, "A stochastic MPC based approach to integrated energy management in microgrids", Sustainable Cities and Society, vol. 41, pp. 349-362, Aug. 2018.

[14] H. Farzin, M. F. Firuzabad, M. M. Aghtaie, "Stochastic energy management of microgrids during unscheduled islanding period", IEEE Transactions on Industrial Informatics, vol. 13, no. 3, pp. 1079-1087, June 2017

[15] B. Zhang, Q. Li, L. Wang, and W. Feng, "Robust optimization for energy transactions in multi-microgrids under uncertainty", Applied Energy, vol. 217, pp. 346-360, 2018

[16] A. Eydeland and K. Wolyniec, Energy and power risk management. Chichester, U.K., Wiley, 2003

[17] D. Wang, K. Meng, C. Coates, X. Gao, Z. Dong, "Coordinated dispatch of networked energy storage systems for loading management in active distribution networks," IET Renewable Power Generation., vol. 10, no. 9, pp. 1374-1381, Oct. 2017.

[18] J. Qiu, J. Zhao, and D. Wang, "Flexible multi-objective transmission expansion planning with adjustable risk aversion", Energies, vol. 10, no. 7, pp. 1025-1036, July 2017.

[19] The MOSEK optimization toolbox for MATLAB manual. Version 7.0 [Online] Available: http://docs.mosek.com/7.0/toolbox/ (Accessed 25/03/2019)

[20] Australia Energy Market Operator, [Online]: https://www.aemo.com.au/ (Accessed 25/03/2019). 